\title{
Effects of recommendations to follow the Dietary Approaches to Stop Hypertension (DASH) diet $v$. usual dietary advice on childhood metabolic syndrome: a randomised cross-over clinical trial
}

\author{
Parvane Saneei $^{1,2}$, Mahin Hashemipour ${ }^{3,4}$, Roya Kelishadi ${ }^{4,5}$, Somayeh Rajaei ${ }^{1,2}$ \\ and Ahmad Esmaillzadeh ${ }^{1,2 *}$ \\ ${ }^{1}$ Food Security Research Center, Isfahan University of Medical Sciences, Isfahan, Iran \\ ${ }^{2}$ Department of Community Nutrition, School of Nutrition and Food Science, Isfahan University of Medical Sciences, \\ PO Box 81745-151, Isfahan, Iran \\ ${ }^{3}$ Department of Pediatric Endocrinology, Child Growth and Development Research Center, Isfahan University of Medical \\ Sciences, Isfahan, Iran \\ ${ }^{4}$ Department of Pediatrics, Faculty of Medicine, Isfahan University of Medical Sciences, Isfahan, Iran \\ ${ }^{5}$ Department of Pediatrics, Child Growth and Development Research Center, Isfahan University of Medical Sciences, \\ Isfahan, Iran
}

(Submitted 5 October 2012 - Final revision received 29 April 2013 - Accepted 29 April 2013 - First published online 18 June 2013)

\begin{abstract}
The effects of the Dietary Approaches to Stop Hypertension (DASH) eating plan on childhood metabolic syndrome (MetS) and insulin resistance remain to be determined. The present study aimed to assess the effects of recommendations to follow the DASH diet $v$. usual dietary advice (UDA) on the MetS and its features in adolescents. In this randomised cross-over clinical trial, sixty post-pubescent adolescent girls with the MetS were randomly assigned to receive either the recommendations to follow the DASH diet or UDA for 6 weeks. After a 4-week washout period, the participants were crossed over to the alternate arm. The DASH group was recommended to consume a diet rich in fruits, vegetables and low-fat dairy products and low in saturated fats, total fats and cholesterol. UDA consisted of general oral advice and written information about healthy food choices based on healthy MyPlate. Compliance was assessed through the quantification of plasma vitamin C levels. In both the groups, fasting venous blood samples were obtained at baseline and at the end of each phase of the intervention. The mean age and weight of the participants were 14.2 (SD 1.7) years and 69 (SD 14.5 ) kg, respectively. Their mean BMI and waist circumference were $27.3 \mathrm{~kg} / \mathrm{m}^{2}$ and $85.6 \mathrm{~cm}$, respectively. Serum vitamin C levels tended to be higher in the DASH phase than in the UDA phase (860 ( $\operatorname{se~104)~v.~} 663$ ( $\operatorname{se~76)~ng/l,~respectively,~} P=0 \cdot 06$ ). Changes in weight, waist circumference and BMI were not significantly different between the two intervention phases. Although changes in systolic blood pressure were not statistically significant between the two groups $(P=0 \cdot 13)$, recommendations to follow the DASH diet prevented the increase in diastolic blood pressure compared with UDA $(P=0 \cdot 01$ ). We found a significant within-group decrease in serum insulin levels (101.4 (SE 6.2) $v$. 90.0 (SE 5.5) pmol/l, respectively, $P=0.04)$ and a non-significant reduction in the homeostasis model assessment for insulin resistance score $(P=0 \cdot 12)$ in the DASH group. Compared with the UDA group, the DASH group experienced a significant reduction in the prevalence of the MetS and high blood pressure. Recommendations to follow the DASH eating pattern for 6 weeks among adolescent girls with the MetS led to reduced prevalence of high blood pressure and the MetS and improved diet quality compared with UDA. This type of healthy diet can be considered as a treatment modality for the MetS and its components in children.
\end{abstract}

Key words: DASH diet: Metabolic syndrome: Children: Obesity

The metabolic syndrome (MetS) is a clustering of cardiovascular risk factors including abdominal obesity, hypertension, abnormal glucose homeostasis and dyslipidaemia ${ }^{(1)}$. This syndrome increases the risk of atherosclerosis, type 2 diabetes and stroke and enhances the mortality rate by $20-80 \%{ }^{(2-5)}$. It seems that insulin resistance is the primary defect in the $\operatorname{MetS}^{(6)}$. Findings from the Third National Health and Nutrition Examination Survey have revealed that the MetS is prevalent among $24 \%$ of men and $23 \%$ of women in the USA ${ }^{(7)}$. In Iran, the MetS is highly prevalent in adults, with an estimated prevalence of

Abbreviations: BP, blood pressure; DASH, Dietary Approaches to Stop Hypertension; DBP, diastolic blood pressure; HOMA-IR, homeostasis model assessment for insulin resistance; MetS, metabolic syndrome; SBP, systolic blood pressure; UDA, usual dietary advice. 
more than $30 \%{ }^{(8,9)}$. In children, there is no general consensus on the cut-off values and components of the MetS ${ }^{(3,10)}$. Based on the modified Adult Treatment Panel III definition, $10 \%$ of Iranian adolescents are affected by the syndrome ${ }^{(11)}$. Since the process of atherosclerosis, which is linked to obesity and other components of the MetS, starts at an early age, the primary prevention and treatment of childhood MetS are of great importance ${ }^{(6)}$.

Lifestyle interventions, including diet therapy, have long been recommended for the management of the MetS ${ }^{(12)}$. In order to prescribe a therapeutic diet, all abnormalities of the MetS should be taken into account ${ }^{(4)}$. Most individuals with the MetS are overweight or obese, and weight loss is the key recommendation to improve all aspects of the $\mathrm{MetS}^{(13)}$. Among children, energy restriction and weight loss can hinder growth and development ${ }^{(2)}$. Therefore, the appropriate diet for the management of childhood MetS is the one that meets the growth requirements of children besides modifying their metabolic abnormalities ${ }^{(2)}$. The Dietary Approaches to Stop Hypertension (DASH) eating plan might be beneficial for patients with the MetS ${ }^{(14)}$. The DASH diet is rich in vegetables, fruits, whole grains, low-fat dairy products, $\mathrm{Mg}, \mathrm{K}, \mathrm{Ca}$ and fibre $^{(15)}$. The DASH diet also has a low Na content $(2400 \mathrm{mg}$ / d) ${ }^{(16)}$. Adherence to the DASH diet has been reported to lower blood pressure ${ }^{(15)}$ and lipid profiles ${ }^{(17)}$. The beneficial effect of the DASH diet on glycaemic control and liver enzymes in type 2 diabetic patients has also been documented ${ }^{(18)}$. Limited data indicating the effects of the DASH diet on the MetS are available. We are aware of just one study that has assessed the effects of the DASH diet on the MetS among adults ${ }^{(14)}$. To our knowledge, the effects of the DASH diet on the features of the MetS in children have not been examined yet. Furthermore, the DASH diet has been applicable and acceptable among the adult population ${ }^{(19)}$, but its acceptability in children remains unknown. The DASH diet contains high amounts of low-fat dairy products, $\mathrm{Ca}, \mathrm{K}$ and $\mathrm{Mg}$, all of which have been shown to affect the features of the MetS $S^{(20,21)}$. Moreover, the restricted $\mathrm{Na}$ content of the DASH diet leads to lower blood pressure levels ${ }^{(15)}$. In addition, greater amounts of dietary fibre, folate, vitamin $\mathrm{C}$, carotenoids, phytosterols, phytochemicals and antioxidants in the DASH diet ${ }^{(22)}$ make it suitable for treating the MetS and maintaining normal growth in children and adolescents. Therefore, the present study was conducted to assess the effects of recommendations to follow the DASH diet $v$. usual dietary advice (UDA) on the MetS and its features in adolescents. We hypothesised that recommendations to follow the DASH diet would be an appropriate approach for the treatment of children with the MetS. Since dietary adherence of females is higher than that of males ${ }^{(23)}$, we conducted the present study among adolescent girls.

\section{Subjects and methods}

\section{Participants}

A total of sixty female post-pubescent adolescent girls aged 11-18 years with the MetS participated in the present study. The MetS was defined as having three or more of the following criteria, according to the Adult Treatment Panel III criteria modified for children and adolescents ${ }^{(24)}$ : (1) abdominal adiposity (waist circumference $>75$ th percentile for age); (2) low levels of serum HDL-cholesterol $(<500 \mathrm{mg} / \mathrm{l})$; (3) hypertriacylglycerolaemia (TAG levels $\geq 1000 \mathrm{mg} / 1$ ); (4) elevated blood pressure (systolic blood pressure (SBP) or diastolic blood pressure (DBP) >90th percentile for age and height from the National Heart, Lung and Blood Institute's recommended cut-off points); (5) impaired glucose homeostasis (fasting plasma glucose levels $\geq 1000 \mathrm{mg} / \mathrm{l}$ ). Subjects were not included in the study if they had CVD or kidney or liver diseases or were taking any medications affecting nutrient metabolism, blood lipids and blood pressure or any vitamin and mineral supplements. The required sample size for the present study was determined using serum TAG levels ${ }^{(24)}$ as a key dependent variable. We used the standard formula suggested for two-period, two-treatment cross-over studies. Given the $80 \%$ power and type I error of $5 \%$, we required a sample size of thirty-two participants to detect significant differences between the two groups. Considering the high dropouts in cross-over trials, we recruited sixty female adolescents based on the inclusion criteria. Parental written consent and adolescent assent were obtained. During the study, eleven subjects were dropped out. The reasons for dropping out were as follows: fear of blood sampling ( $n$ 6); educationally busy ( $n$ 2); surgery ( $n$ 1); personal reasons ( $n$ 1); mother's demise ( $n$ 1). Finally, forty-nine adolescent girls completed the whole trial. The present study was conducted according to the guidelines laid down in the Declaration of Helsinki. The study was ethically approved by the Food Security Research Center of Isfahan University of Medical Sciences and has been registered at the Iranian registry of clinical trials (http://www.irct.ir; identifier: IRCT201110191485N6).

\section{Study design}

In this cross-over randomised clinical trial, after obtaining detailed information about their lifestyle characteristics in a run-in period of 2 weeks, the participants were randomly allocated to follow the DASH diet or UDA for 6 weeks. Random assignment was done by the use of computer-generated random numbers. A 4-week washout period separated the two phases of the study. During this period, the participants were requested to go back to their old eating habits. After the washout period, the participants were crossed over to the alternate arm: those consuming the DASH diet in the first phase were asked to follow UDA and vice versa. Except for the participants and the study dietitian who provided dietary advice, all study personnel (including those working in the laboratories) and other researchers were blinded to the dietary assignment. Each participant completed a $3 \mathrm{~d}$ dietary record (two week days and one weekend day) during the run-in period as well as throughout each phase of the intervention. Food intake was recorded by the participant at the time the foods were consumed to minimise reliance on memory. To assess compliance to the DASH diet, the average of the $3 \mathrm{~d}$ dietary records obtained from the participants in the DASH phase was used. Food diaries were analysed for their nutrient content using the Nutritionist-IV software 
(First Databank), which was modified for Iranian foods. Compliance to the DASH diet was also assessed using serum vitamin C levels as well as dietary records. The participants were asked to not change their habitual physical activity levels throughout the study. They were asked to record their physical activities for $2 \mathrm{~d}$ in the run-in period and throughout each phase of the intervention.

\section{The Dietary Approaches to Stop Hypertension diet}

The DASH diet used in this intervention was slightly modified from the original version of the DASH diet for adults to more closely conform to the unique nutritional needs of adolescents. Energy requirements of each participant were calculated individually based on equations suggested by the American Dietetic Association, Pediatric Weight Management Guidelines for weight maintenance ${ }^{(25)}$. We did not prescribe weight loss diets for adolescents. All diets were designed to maintain the current weight. Diets were prescribed according to the energy requirements of the adolescents. The macronutrient composition of the DASH diet was as follows: carbohydrates, 53-58\%; proteins, 15-18\%; total fats, $26-30 \%$ of total energy. The dietary goals of the DASH group as well as a sample of the $1 \mathrm{~d}$ menu of the DASH diet are summarised in Table 1. The DASH diet was planed to contain high amounts of whole grains, fruits, vegetables and low-fat dairy products as well as low amounts of saturated fats, cholesterol, refined grains, sweets and red meat. $\mathrm{Ca}, \mathrm{K}$ and $\mathrm{Mg}$ contents of the DASH diet were higher than those recommended during UDA. The DASH diet contained $<2400 \mathrm{mg} \mathrm{Na} / \mathrm{d}$. Both groups receiving the DASH recommendations and UDA were visited monthly; each session for a patient took about $30-45 \mathrm{~min}$. In the sessions, the study nutritionist explained the benefits of the diet to the participants. The participants also received the required information to use the exchange list of foods. They were taught as to how to record their dietary intakes. A $7 \mathrm{~d}$ menu cycle based on the energy requirements of each participant was planned. Moreover, the participants were in touch with the nutritionist by phone every day. The dietitian made calls to the participants to remind them to record their food intakes and physical activities and to ask them to follow the prescribed diet. The dietitian also asked the participants if there was any problem with the diet. She also responded to the questions that the participants had about the diet. The participants were also free to make calls to the dietitian whenever they had questions regarding their diet.

\section{Usual dietary advice}

The UDA group was given general oral and written information about healthy food choices based on healthy MyPlate ${ }^{(26)}$. The eating patterns of the group were similar to that of a typical Iranian diet (carbohydrates, 50-60\%; proteins, $15-20 \%$; total fats, $<30 \%$; dietary fibre, $14 \mathrm{~g} / \mathrm{d}$; SFA, $\left.24 \mathrm{~g} / \mathrm{d}^{(27)}\right)$. Ca, dairy product, nut and legume contents of this diet were lower than those of the DASH diet. No dietary menus were provided for this group.

Table 1. Dietary goals of the Dietary Approaches to Stop Hypertension (DASH) intervention $v$. usual dietary advice and a sample menu of the DASH diet

\begin{tabular}{|c|c|c|}
\hline DASH goals & & A sample of usual dietary advice \\
\hline $\begin{array}{l}\text { The DASH intervention includes at least eight servings } / \mathrm{d} \text { of fruits } \\
\text { and vegetables, two to three servings } / \mathrm{d} \text { of low-fat dairy foods, } \\
30 \mathrm{~g} / \mathrm{d} \text { of red meat and }<2400 \mathrm{mg} / \mathrm{d} \text { of } \mathrm{Na}\end{array}$ & & $\begin{array}{l}\text { Usual dietary advice consisted of healthy food choices based } \\
\text { on healthy My Plate (see reference } 26 \text { ) }\end{array}$ \\
\hline A sample of the DASH diet & & Eat slowly and chew food well and enjoy your food \\
\hline Energy (kcal) & 1500 & Steam, boil and grill foods instead of frying them \\
\hline Energy $(\mathrm{kJ})$ & 6276 & Remove fats and skin of the chicken and meat before cooking \\
\hline Servings/d & & $\begin{array}{l}\text { Try to use whole-wheat and barley breads instead of rice and } \\
\text { pasta }\end{array}$ \\
\hline Grains & 6 & Do not skip any meals \\
\hline Vegetables & 5 & Eat frequently while keeping the amount constant \\
\hline Fruits & 4 & $\begin{array}{l}\text { Minimise the intake of sugar, sweets, cakes, cookies } \\
\text { and sweetened drinks }\end{array}$ \\
\hline Dairy products & 2.5 & Try to have a variety of foods in your daily diet \\
\hline Meats and alternatives & 2.5 & \\
\hline Nuts/seeds & 1 & \\
\hline Fats and oils & 6 & \\
\hline
\end{tabular}

A menu sample of the DASH diet (6276 kJ (1500 kcal), 55\% carbohydrate, $17 \%$ protein, $28 \%$ fats, $3312 \mathrm{mg} \mathrm{K}, 1357 \mathrm{mg} \mathrm{Ca}, 458 \mathrm{mg} \mathrm{Mg}$ and $2347 \mathrm{mg} \mathrm{Na}$ )

Breakfast: two slices of whole-wheat bread $(60 \mathrm{~g})$ and one egg-white

Snack 1: one medium orange and one medium apple

Lunch: ten tablespoons of cooked rice and stew made with 1/2 cup of green bean, onion, tomato and carrot, $30 \mathrm{~g}$ of red meat, three teaspoons of rapeseed oil, 1/2 cup of low-fat yogurt, salad made with two cups of fresh lettuce, green pepper, cucumber and one teaspoon of lemon juice Snack 2: one cup of low-fat milk, one date and two walnuts

Snack 3: one cup of tea, one date and 1/2 cup of pomegranate

Dinner: two slices of whole-wheat bread $(60 \mathrm{~g}), 30 \mathrm{~g}$ of chicken, one cup of cooked vegetables (mushroom, onion, tomato and carrot) and two teaspoons of rapeseed oil

Snack 4: one cup of low-fat milk and one date 


\section{Measurements}

Body weight was measured while the participants were minimally clothed and without shoes, using digital scales, and it was recorded to the nearest $0 \cdot 1 \mathrm{~kg}$. Height was measured in a standing position and without shoes, using a tape meter while the shoulders were in a normal position. BMI was calculated as weight (in $\mathrm{kg}$ ) divided by height (in $\mathrm{m}^{2}$ ). Waist circumference was measured to the nearest $0 \cdot 1 \mathrm{~cm}$ at the narrowest level over light clothing, using an unstretched tape meter, without any pressure to the body surface. Data on physical activities were collected through physical activity records. The participants were taught at the run-in period (before the start of the intervention) as to how to record these data. They were requested to record the type of physical activity and its intensity as well as the duration of that activity. The sum of the durations of activities in the physical activity records had to be $24 \mathrm{~h}$. In case of any problem in recording, the dietitian contacted the participants to further review the type, intensity and duration of the activities recorded in the records. Data from the physical activity records are expressed as metabolic equivalents. Blood pressure was measured twice after the participants sat for 15 min using a standard digital sphygmomanometer (OMRON, M2; HEM.7117-E) with an appropriate cuff size. Overall, two blood pressure measurements were made at a 5 min interval, and the mean of the two measurements was used for the analysis. To avoid subjective errors, all measurements were made by the same nutritionist.

Fasting blood samples for the measurement of glucose and lipid concentrations were drawn after an overnight fast of $12 \mathrm{~h}$. Fasting blood glucose concentration was measured on the day of blood collection using the enzymatic colorimetric method with glucose oxidase. Serum concentrations of total cholesterol and TAG were measured using commercially available enzymatic reagents (Pars Azmoon). Serum HDL-cholesterol levels were measured after precipitation of the apoB-containing lipoproteins with phosphotungstic acid. LDL-cholesterol levels were calculated according to the Friedewald equation. Inter-assay and intra-assay CV were 0.9 and $1.1 \%$ for total cholesterol and 1.6 and $1.2 \%$ for TAG, respectively. Serum insulin concentrations were measured using ELISA kits and an ELISA reader (Diagnostic Biochem Canada Inc.). Insulin resistance was estimated on the basis of fasting glucose and insulin concentrations using the homeostasis model assessment for insulin resistance (HOMA-IR) method. Plasma vita- min C levels were measured using commercially available kits (Glory Science Company) by the ELISA method.

\section{Statistical analysis}

To ensure the normal distribution of variables, we used the Kolmogorov-Smirnov test. Log transformation was applied for non-normally distributed variables. The analyses were carried out based on the intention-to-treat approach. Missing values were treated based on the last-observation-carriedforward method. Descriptive statistics (means, SEM or SD and range) for general characteristics of the study participants are reported. Data on dietary intakes and physical activities were compared by a paired $t$ test. For each dependent variable, we computed the changes from baseline by subtracting the baseline value from the end-of-trial value. Within-group and between-group changes in anthropometric measures as well as biochemical indicators were compared using a paired-samples $t$ test. Differences in the prevalence of the MetS and its components were examined using the $\chi^{2}$ test. We also assessed whether the carry-over effect was significant. To assess the carry-over effect, we computed the average of end-of-trial values for each variable for two treatments separately and compared the means of the two treatment orders using a $t$ test. $P$ values $<0.05$ were considered as significant.

\section{Results}

General characteristics of the study population are given in Table 2. The mean age and weight of the participants were 14.2 years and $69 \mathrm{~kg}$, respectively. Their mean BMI and waist circumference were $27.3 \mathrm{~kg} / \mathrm{m}^{2}$ and $85.6 \mathrm{~cm}$, respectively. At baseline, mean SBP and DBP of the participants were 120.6 and $73.3 \mathrm{mmHg}$.

The average energy intake of the participants during the run-in period, based on $3 \mathrm{~d}$ dietary records, was $7510 \mathrm{~kJ} / \mathrm{d}$ $(1795 \mathrm{kcal} / \mathrm{d})$. The mean dietary intakes of grains, fruits and vegetables were $7 \cdot 6,2 \cdot 4$ and $2 \cdot 2$ servings/d, respectively. The mean intakes of dairy products, meat and alternatives, and fats were 1, 4.2 and $10 \cdot 2$ servings/d, respectively. Dietary energy density was $4.6 \mathrm{~kJ} / \mathrm{g}(1.1 \mathrm{kcal} / \mathrm{g})$. The participants obtained $13 \%$ of their energy from proteins, $50 \%$ from carbohydrates and $37 \%$ from fats. Mean Na and $\mathrm{K}$ intakes were 2218 and $2481 \mathrm{mg} / \mathrm{d}$, respectively.

Compliance to the DASH diet was assessed through dietary records as well as quantification of serum vitamin C levels.

Table 2. Baseline characteristics of the study participants

(Mean values and standard deviations; minimum and maximum values; ranges)

\begin{tabular}{|c|c|c|c|c|c|}
\hline & Mean & SD & Minimum & Maximum & Range \\
\hline Age (years) & $14 \cdot 2$ & $1 \cdot 7$ & $9 \cdot 8$ & $17 \cdot 9$ & $9 \cdot 8-17 \cdot 9$ \\
\hline Weight (kg) & $69 \cdot 0$ & 14.5 & $40 \cdot 7$ & $103 \cdot 4$ & $40 \cdot 7-103 \cdot 4$ \\
\hline Height (cm) & $158 \cdot 3$ & $6 \cdot 3$ & $140 \cdot 0$ & $172 \cdot 0$ & $140 \cdot 0-172 \cdot 0$ \\
\hline BMI $\left(\mathrm{kg} / \mathrm{m}^{2}\right)$ & $27 \cdot 3$ & $4 \cdot 1$ & $20 \cdot 1$ & $37 \cdot 2$ & $20 \cdot 1-37 \cdot 2$ \\
\hline Weight circumference $(\mathrm{cm})$ & $85 \cdot 6$ & 8.9 & 66.5 & $109 \cdot 0$ & $66 \cdot 5-109 \cdot 0$ \\
\hline Systolic blood pressure $(\mathrm{mmHg})$ & $120 \cdot 6$ & $8 \cdot 7$ & $100 \cdot 0$ & $140 \cdot 0$ & $100 \cdot 0-140 \cdot 0$ \\
\hline Diastolic blood pressure $(\mathrm{mmHg})$ & 73.3 & $10 \cdot 9$ & $49 \cdot 0$ & $110 \cdot 0$ & $49 \cdot 0-110 \cdot 0$ \\
\hline
\end{tabular}


The energy-adjusted dietary intakes of the participants throughout the run-in period and the intervention phases are shown in Fig. 1. Mean dietary energy intakes were not significantly different between the two groups during the intervention (DASH: $6788.1 \quad$ (SE $269 \cdot 4) \mathrm{kJ} / \mathrm{d} \quad(1622.4$ $(\mathrm{SE} 64.4) \mathrm{kcal} / \mathrm{d}) \quad v$. UDA: $7078.1(\mathrm{SE} 297.5) \mathrm{kJ} / \mathrm{d}(1691.7$ (SE $71 \cdot 1) \mathrm{kcal} / \mathrm{d}$ ), $P=0 \cdot 31$ ). During the DASH phase, the participants consumed higher amounts of carbohydrates, Ca, K and dietary fibre and lower amounts of fats, saturated fats and Na compared with the UDA phase (all $P$ values $<0.05$ ). The participants also tended to consume higher amounts of fruits and vegetables $(P=0.06)$ and vitamin $C(P=0.08)$ in the DASH phase than in the UDA phase. Dietary energy density was significantly lower during the DASH phase than during the UDA phase $(4 \cdot 1$ (SE 0.0) v. $5 \cdot 0(\operatorname{SE} 0 \cdot 0) \mathrm{kJ} / \mathrm{g}(1 \cdot 0$ (SE $0 \cdot 0) v \cdot 1 \cdot 2(\operatorname{SE~} 0 \cdot 0) \mathrm{kcal} / \mathrm{g}$ ), respectively, $P=0 \cdot 01)$. Serum vitamin $\mathrm{C}$ levels also tended to be higher in the DASH phase than in the UDA phase (860 (SE 104) v. 663 (SE 76) ng/l, respectively, $P=0.06$ ). The change in vitamin $\mathrm{C}$ levels in the DASH phase was 168 (SE 129) and it was - 138 (SE 97) ng/l in the UDA phase. Comparison of the changes between the two groups revealed a borderline significant difference $(P=0.06)$. All these data indicate a relatively good compliance of the participants to the DASH eating plan.

Findings from the physical activity records revealed that the activity levels of the participants were not significantly different when comparing the two intervention phases (mean physical activity in the DASH phase: 32.8 (SE 0.5) metabolic equivalents- $\mathrm{h} / \mathrm{d}$ and that in the UDA phase: 32.7 (SE $0 \cdot 4$ ) metabolic equivalents-h; $P=0 \cdot 85$ )

The effects of recommendations to follow the DASH diet $v$. UDA on anthropometric measures and blood pressure (BP) are summarised in Table 3. Recommendations to follow the DASH diet resulted in a significant reduction in BMI and waist circumference, while UDA led to a significant decrease in waist circumference only. However, changes in weight, waist circumference and BMI were not significantly different between the two phases. Recommendations to follow the DASH diet did not considerably influence SBP $(P=0.82)$ and DBP $(P=0 \cdot 21)$, whereas during the UDA phase, the participants experienced a significant increase in SBP $(P=0.04)$ and DBP $(P=0 \cdot 01)$. Although changes in SBP between the two phases were not statistically significant $(P=0 \cdot 13)$, the alterations in DBP were significant $(P=0 \cdot 01)$; that is, recommendations to follow the DASH diet prevented the increase in DBP compared with the usual recommendations.

The effects of recommendations to follow the DASH diet on the features of the MetS are given in Table 4. Plasma concentrations of fasting blood glucose and serum lipid profiles were not affected by the recommendations to follow the DASH diet. However, these recommendations resulted in a significant decrease in serum insulin levels $(P=0.04)$ and a non-significant reduction in the HOMA-IR score $(P=0 \cdot 12)$. However, when we compared the changes in fasting blood glucose, serum lipid and insulin levels and HOMA-IR score between the two phases, we failed to find a significant difference.

The effects of recommendations to follow the DASH diet on the prevalence of the MetS and its components are presented in Table 5. Despite a slight decline in the prevalence of the MetS and its features, we failed to find a significant effect of recommendations to follow the DASH diet on most of its features. Compared with UDA, recommendations to follow the DASH diet resulted in a significant reduction in the prevalence of the MetS and high blood pressure.

For most MetS-related variables, we found no evidence of a carry-over effect, except for DBP, for which we observed a significant carry-over effect from period 1 to period $2(P=0 \cdot 03)$.

\section{Discussion}

In the present randomised cross-over clinical trial, we found that recommendations to follow the DASH diet among children, compared with UDA, resulted in improved diet quality and reduced the prevalence of the MetS and high blood pressure. No other significant effects were observed for the recommendations to follow the DASH diet compared with UDA. To our knowledge, the present study is the first one to examine the effects of recommendations to follow the DASH diet on the MetS and its features in adolescents.

Along with increasing prevalence of childhood obesity, the prevalence of the MetS is rising among children and adolescents. This syndrome would in turn lead to increased rates of type 2 diabetes mellitus and CVD among youths. Therefore, the management of childhood MetS is an important priority for health care systems at individual and population levels. Weight reduction is the cornerstone in the management of the MetS; however, weight loss is generally not recommended for children since childhood is a period of rapid growth and development. The suitable dietary approach for childhood MetS is still a matter of discussion mainly due to scarcity of data. We hypothesised the DASH dietary pattern to be a suitable choice for improving the features of childhood MetS that could maintain the weight of children and provide adequate nutrients for their growth and development. In the present study, compared with UDA for childhood MetS, recommendations to follow the DASH diet resulted in significant reductions in dietary energy density and intakes of saturated fats and $\mathrm{Na}$ along with increased intakes of $\mathrm{K}$, Ca, dietary fibre, fruits, vegetables and vitamin $\mathrm{C}$.

In the present study, we observed a significant favourable effect of recommendations to follow the DASH diet on the prevalence of the MetS and high BP. This finding is in line with those of earlier studies on adolescents that have reported the beneficial effects of the components of the DASH diet on the MetS. The results of the National Health and Nutrition Examination Survey 1999-2002 revealed that the Healthy Eating Index and fruit scores were associated with a lower prevalence of the MetS in US adolescents ${ }^{(28)}$. Fibre-rich, nutrient-dense, plant-based foods have also been inversely associated with the prevalence of childhood $\mathrm{MetS}^{(29)}$. In a cross-sectional study among 2130 youths with type 1 diabetes, Liese et $a l .{ }^{(30)}$ found that the greater adherence to the DASH diet was inversely associated with the LDL:HDL ratio and glycated $\mathrm{Hb}$. However, they failed to find significant associations with serum TAG and LDL-cholesterol levels. 

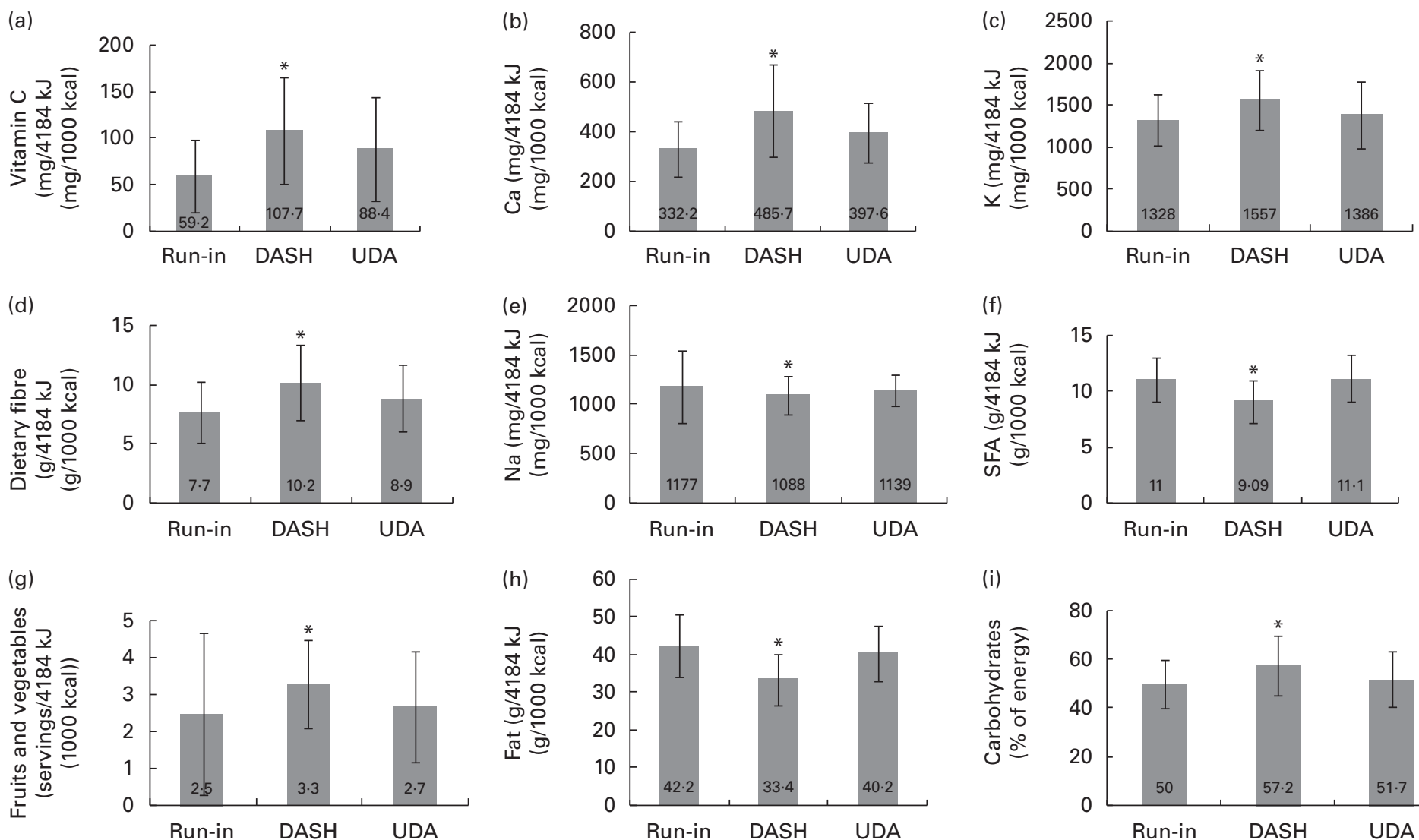

(i)

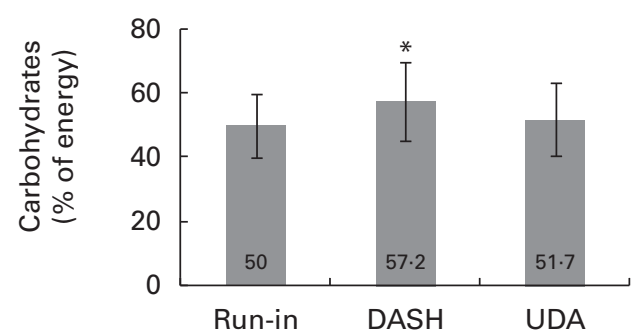

Fig. 1. Energy-adjusted dietary intakes of the participants throughout the run-in period and the 6-week intervention. The Dietary Approaches to Stop Hypertension (DASH) diet was high in fruits, vegetables, whole grains and low-fat dairy products as well as low in saturated fats, total fats, cholesterol, refined grains and sweets ((a) vitamin C, (b) calcium, (c) potassium, (d) dietary fibre, (e) sodium, (f) SFA, (g) fruits and vegetables, (h) fats and (i) carbohydrates). The usual dietary advice (UDA) group received general oral and written information about healthy food choices, and the intakes of the group were as those for the usual Iranian diet (carbohydrates, $50-60 \%$; proteins, $15-20 \%$; total fats, $<30 \%$ ). Values are means, with standard errors (given within each bar) represented by vertical bars. ${ }^{*}$ Mean values were significantly different from those of the run-in and UDA phases: (a) $P=0.08$, (b) $P=0.003$, (c) $P=0.02$, (d) $P=0.03$, (e) $P=0.12$, (f) $P<0.001$, (g) $P=0.06$, (h) $P<0.001$ and (i) $P=0.01$ (paired $t$ test) 
Table 3. Effects of recommendations to follow the Dietary Approaches to Stop Hypertension (DASH) diet $v$. usual dietary advice on anthropometric measures and blood pressure of children with the metabolic syndrome

(Mean values with their standard errors)

\begin{tabular}{|c|c|c|c|c|c|c|c|c|c|c|c|c|c|c|c|}
\hline & \multicolumn{7}{|c|}{ DASH $\operatorname{diet}^{*}$} & \multicolumn{7}{|c|}{ Usual dietary advice† } & \multirow[b]{3}{*}{$P \|$} \\
\hline & \multicolumn{2}{|c|}{ Baseline } & \multicolumn{2}{|c|}{ 6th week } & \multicolumn{2}{|c|}{ Change } & \multirow[b]{2}{*}{$P \S$} & \multicolumn{2}{|c|}{ Baseline } & \multicolumn{2}{|c|}{ 6th week } & \multicolumn{2}{|c|}{ Changef } & \multirow[b]{2}{*}{$P \S$} & \\
\hline & Mean & SEM & Mean & SEM & Mean & SEM & & Mean & SEM & Mean & SEM & Mean & SEM & & \\
\hline Weight (kg) & 68.5 & 1.8 & $68 \cdot 3$ & 1.9 & -0.16 & $0 \cdot 18$ & 0.38 & $68 \cdot 7$ & 1.9 & 68.6 & 1.9 & -0.14 & 0.20 & 0.49 & 0.94 \\
\hline BMI $\left(\mathrm{kg} / \mathrm{m}^{2}\right)$ & $27 \cdot 1$ & 0.5 & $26 \cdot 8$ & 0.5 & -0.28 & 0.08 & $<0.001$ & $27 \cdot 1$ & 0.5 & $26 \cdot 9$ & 0.5 & -0.14 & 0.08 & 0.07 & 0.17 \\
\hline Waist circumference (cm) & 84.5 & $1 \cdot 1$ & $82 \cdot 7$ & $1 \cdot 2$ & -1.77 & 0.33 & $<0.001$ & $85 \cdot 0$ & $1 \cdot 2$ & 83.8 & 1.2 & $-1 \cdot 18$ & 0.36 & $<0.001$ & 0.23 \\
\hline Systolic blood pressure (mmHg) & $118 \cdot 8$ & 1.3 & 118.5 & 1.2 & -0.25 & $1 \cdot 12$ & 0.82 & 117.5 & 1.3 & $120 \cdot 0$ & 1.2 & 2.51 & $1 \cdot 19$ & 0.04 & 0.13 \\
\hline Diastolic blood pressure $(\mathrm{mmHg})$ & 73.9 & $1 \cdot 1$ & 72.5 & 0.9 & -1.08 & 0.85 & 0.21 & $71 \cdot 3$ & 1.2 & 74.4 & 1.0 & $3 \cdot 11$ & 1.08 & 0.01 & 0.01 \\
\hline
\end{tabular}

* The DASH diet was high in fruits, vegetables, whole grains and low-fat dairy products and low in saturated fats, total fats, cholesterol, refined grains and sweets. The amount of $\mathrm{Na}$ intake was $2400 \mathrm{mg} / \mathrm{d}$.

†The usual dietary advice group received general oral and written information about healthy food choices, and the intakes of the group were as those for the usual Iranian diet (carbohydrates, $50-60 \%$; proteins, $15-20 \%$; total fats, $<30 \%$.

$\ddagger$ Calculated by subtracting the values at the 6th week from the values at baseline.

$\S$ For comparison of within-group differences by a paired $t$ test.

Table 4. Effects of recommendations to follow the Dietary Approaches to Stop Hypertension (DASH) diet $v$. usual dietary advice on metabolic profiles in the participants (Mean values with their standard errors)

\begin{tabular}{|c|c|c|c|c|c|c|c|c|c|c|c|c|c|c|c|}
\hline & \multicolumn{7}{|c|}{ DASH $\operatorname{diet}^{\star}$} & \multicolumn{7}{|c|}{ Usual dietary advice† } & \multirow[b]{3}{*}{$P \|$} \\
\hline & \multicolumn{2}{|c|}{ Baseline } & \multicolumn{2}{|c|}{ 6th week } & \multicolumn{2}{|c|}{ Changeł } & \multirow[b]{2}{*}{$P \S$} & \multicolumn{2}{|c|}{ Baseline } & \multicolumn{2}{|c|}{6 th week } & \multicolumn{2}{|c|}{ Changeł } & \multirow[b]{2}{*}{$P \S$} & \\
\hline & Mean & SEM & Mean & SEM & Mean & SEM & & Mean & SEM & Mean & SEM & Mean & SEM & & \\
\hline Fasting plasma glucose (mg/l) & 908 & 17 & 913 & 9 & 5 & 12 & 0.65 & 908 & 10 & 909 & 8 & $2 \cdot 0$ & 8.4 & 0.81 & 0.82 \\
\hline Serum TAG $(\mathrm{mg} / \mathrm{l})$ & 977 & 53 & 969 & 56 & $-8 \cdot 2$ & 38.8 & 0.83 & 972 & 56 & 956 & 52 & $-15 \cdot 5$ & 34.7 & 0.66 & 0.90 \\
\hline Total cholesterol (mg/l) & 1579 & 30 & 1542 & 29 & -38.0 & $26 \cdot 1$ & 0.15 & 1555 & 26 & 1549 & 28 & $-5 \cdot 3$ & $15 \cdot 9$ & 0.74 & 0.31 \\
\hline HDL-C (mg/l) & 440 & $9 \cdot 0$ & 437 & $9 \cdot 0$ & -3.8 & 5.4 & 0.48 & 427 & 10 & 433 & 10 & 6.3 & 6.5 & 0.33 & 0.31 \\
\hline LDL-C (mg/l) & 884 & 23 & 859 & 19 & $-24 \cdot 3$ & $15 \cdot 8$ & 0.13 & 868 & 18 & 863 & 21 & $-5 \cdot 3$ & $10 \cdot 2$ & 0.60 & 0.32 \\
\hline Insulin (pmol/l) & $101 \cdot 4$ & $6 \cdot 25$ & 90.9 & 1.39 & -1.94 & 1.25 & 0.04 & $20 \cdot 8$ & 1.39 & 20.8 & 1.39 & -0.28 & 1.32 & 0.99 & 0.26 \\
\hline HOMA-IR & 3.3 & 0.2 & 2.9 & 0.2 & -0.28 & 0.18 & 0.12 & $3 \cdot 0$ & 0.2 & 3.0 & 0.2 & -0.04 & 0.19 & 0.86 & 0.36 \\
\hline
\end{tabular}

HDL-C, HDL-cholesterol; LDL-C, LDL-cholesterol; HOMA-IR, homeostasis model assessment for insulin resistance.

*The DASH diet was high in fruits, vegetables, whole grains and low-fat dairy products and low in saturated fats, total fats, cholesterol, refined grains and sweets. The amount of $\mathrm{Na}$ intake was $2400 \mathrm{mg} / \mathrm{d}$.

†The usual dietary advice group received general oral and written information about healthy food choices, and the intakes of the group were as those for the usual Iranian diet (carbohydrates, $50-60 \%$; proteins,

$15-20 \%$; total fats, $<30 \%$ )

¥Calculated by subtracting the values at the 6 th week from the values at baseline.

$\S$ For comparison of within-group differences by a paired $t$ test.

$\|$ For comparison of between-group differences by a paired $t$ test. 
Table 5. Effects of recommendations to follow the Dietary Approaches to Stop Hypertension (DASH) diet $v$. usual dietary advice on the prevalence of the metabolic syndrome and its components in the participants

\begin{tabular}{|c|c|c|c|c|c|c|c|}
\hline & \multicolumn{3}{|c|}{ DASH $\operatorname{diet}^{*}$} & \multicolumn{3}{|c|}{ Usual dietary advice† } & \multirow[b]{2}{*}{$P \S$} \\
\hline & Baseline & 6th week & $P \ddagger$ & Baseline & 6th week & $P \ddagger$ & \\
\hline High WC (\%) & 91.07 & 83.03 & 0.19 & 90 & 85 & 0.40 & 0.43 \\
\hline High FPG (\%) & 15 & 15 & 0.99 & 10 & $8 \cdot 3$ & 0.74 & 0.85 \\
\hline High TAG (\%) & $41 \cdot 7$ & 35 & 0.45 & 40 & 35 & 0.56 & 0.93 \\
\hline Low HDL-C (\%) & 80 & $78 \cdot 3$ & 0.81 & 80 & 80 & 0.99 & 0.63 \\
\hline High BP (\%) & 35 & 30 & 0.56 & 30 & 43.03 & 0.13 & 0.04 \\
\hline MetS (\%) & $56 \cdot 7$ & $46 \cdot 7$ & 0.27 & $53 \cdot 3$ & 53.3 & 0.99 & 0.04 \\
\hline
\end{tabular}

High WC, waist circumference $>75$ th percentile for age; high FPG, fasting plasma glucose levels $\geq 1000 \mathrm{mg} / \mathrm{l}$; high TAG, serum TAG levels $\geq 1000 \mathrm{mg} /$; low HDL-C, serum HDL-cholesterol levels $<500 \mathrm{mg} /$; high $\mathrm{BP}$, systolic or diastolic blood pressure $>90$ th percentile for age and height from the National Heart, Lung and Blood Institute's recommended cut-off point; MetS: metabolic syndrome was defined as having three or more of the above-mentioned criteria, according to the Adult Treatment Panel III criteria modified for children and adolescents.

* The DASH diet was high in fruits, vegetables, whole grains and low-fat dairy products and low in saturated fats, total fats, cholesterol, refined grains and sweets. The amount of $\mathrm{Na}$ intake was $2400 \mathrm{mg} / \mathrm{d}$.

†The usual dietary advice group received general oral and written information about healthy food choices, and the intakes of the group were as those for the usual Iranian diet (carbohydrates, 50-60\%; proteins, 15-20\%; total fats, $<30 \%)$.

¥ For comparison of within-group differences by the $\chi^{2}$ test

$\S$ For comparison of between-group differences by the $\chi^{2}$ test.

In the present study, recommendations to follow the DASH diet, compared with UDA, prevented the rise in DBP, but did not affect SBP. This finding is in contrast to those of the previous studies that reported the significant effect of the DASH eating pattern on SBP, not on DBP, among children ${ }^{(22,31)}$. This difference in findings might be explained by study methodologies. Both the mentioned studies used a parallel design and their participants were pre-hypertensive, hypertensive or healthy children. In a parallel study among adults with the MetS, Azadbakht et al. ${ }^{(14)}$ demonstrated a significant improvement in all the features of the MetS after a 6-month intervention with the DASH diet. The major difference between the present study and that of Azadbakht et al. ${ }^{(14)}$ is that we did not prescribe weight loss diets for adolescents, while they prescribed a weight-reducing DASH diet. In another study, adherence to the DASH diet by adult patients with type 2 diabetes led to a significant reduction in body weight, waist circumference, fasting blood glucose, glycated $\mathrm{Hb}$, LDL-cholesterol levels, and both SBP and DBP after 8 weeks. Although this study had a cross-over design, significant weight reduction of diabetic patients might be responsible for metabolic improvements ${ }^{(32)}$. Furthermore, we found a reasonable acceptability of the DASH diet by adolescents in the present study; however, due to the age group of the study participants, the dietary adherence might not be perfect. Better adherence might have resulted in significant reductions in glucose and lipid profiles in earlier studies among adults. As the adherence to the DASH diet was not completely achieved in the present study, one might not attribute the effects to the changes in nutrient intakes. There is the possibility that the observed effects on blood pressure and the overall MetS may have been due to the within-group significant reduction in BMI and waist circumference in the DASH diet group. It must be noted that the two groups were not significantly different in terms of dependent variables at study baseline. Although the participants met the criteria for the MetS, they were not on the upper end of abnormal cut-points for the features of the MetS, which might reduce the chance of achieving significant changes in these features following the intervention. For example, mean baseline fasting plasma glucose values were less than $900 \mathrm{mg} / \mathrm{l}$ and almost $90 \%$ of the participants had normal values. Moreover, dietary intakes of the participants in the run-in period revealed that they were eating a relatively nutrient-dense diet with fairly high amounts of fruits and vegetables. Their diet had a high content of $\mathrm{K}$ as well as a low content of $\mathrm{Na}$, which might contribute to the non-significant changes in the dependent variables after the intervention.

The mechanisms through which the DASH diet affects metabolic health are largely unknown and still a matter for research. Several possibilities have been suggested: the high contents of $\mathrm{K}, \mathrm{Mg}$ and fibre in the DASH eating pattern may explain the beneficial effects on metabolic profiles ${ }^{(33,34)}$. The low $\mathrm{Na}$ content in this diet can also explain the improvements in $\mathrm{BP}^{(35)}$. While controversial ${ }^{(36)}$, Ca might also have modest BP-lowering effects ${ }^{(37)}$. Higher intakes of legumes ${ }^{(9)}$ and dairy products ${ }^{(20)}$ and lower intakes of saturated fats ${ }^{(38)}$ can account for the beneficial effects of the DASH diet on metabolic parameters. In the present trial, vitamin $\mathrm{C}$ intake also tended to be higher in the DASH group than in the UDA group. Dietary intake of vitamin $\mathrm{C}$ has been suggested to lower BP by enhancing NO synthase activity ${ }^{(39,40)}$. Estimated folate consumption was also greater in the DASH group than in the UDA group. Several studies have indicated the lipid- and BP-lowering effects of folate ${ }^{(41,42)}$. Furthermore, as the DASH diet contains lower amounts of refined sugar, this would in turn help to prevent metabolic abnormalities ${ }^{(43)}$. Most probably, the DASH diet exerts its beneficial effects through a combination of these factors and not just by a single dietary factor ${ }^{(21)}$

The present findings must be interpreted in the context of some limitations. We used serum vitamin $\mathrm{C}$ levels and dietary records to assess the dietary compliance of adolescents. These 
are not perfect tools for assessing the adherence to the DASH diet. Having the cycle menus available might influence the food records and the participants might record what they knew they were supposed to consume based on their cycle menus. Finding a suitable biomarker for adherence to dietary patterns, in particular, to the DASH diet, can be considered in future investigations. We confined the study participants to girls; therefore, the findings cannot be extrapolated to all adolescents. Furthermore, since the Iranian food composition database was not complete, we had to use the USDA food composition database, which encompasses some enriched foods and might overestimate vitamin $\mathrm{C}$, Fe and dietary fibre contents for Iranian foods. In addition, in the present study, the participants were given recommendations to follow the DASH diet (rather than being fed the diet). This might result in imperfect adherence to the DASH diet. Non-achievement of all the DASH goals by our participants (such as $15-16 \mathrm{~g}$ of dietary fibre $/ 4184 \mathrm{~kJ}(1000 \mathrm{kcal}))$ might contribute to the non-significant changes in dependent variables.

In conclusion, recommendations to follow the DASH diet for 6 weeks among adolescent girls with the MetS, compared with UDA, reduced the prevalence of high blood pressure and the MetS and improved diet quality. No significant changes were found in glucose and lipid profiles. This type of healthy diet can be considered as a treatment modality for the MetS and its components in children.

\section{Acknowledgements}

The authors appreciate the Research Council of the Food Security Research Center, Isfahan University of Medical Sciences, for financial support provided for the present study.

The authors' contributions are as follows: P. S. and A. E. contributed to conception, design, statistical analysis and manuscript drafting. P. S. and S. R. contributed to the data collection. M. H. and R. K. contributed to the data collection and manuscript drafting. A. E. supervised the study. All authors approved the final manuscript for submission. A. E. is the guarantor of the work. None of the authors has any personal or financial conflicts of interest.

\section{References}

1. De Ferranti SD \& Osganian SK (2007) Epidemiology of pediatric metabolic syndrome and type 2 diabetes mellitus. Diab Vasc Dis Res 4, 285-296.

2. Halpern A, Mancini MC, Magalhaes ME, et al. (2010) Metabolic syndrome, dyslipidemia, hypertension and type 2 diabetes in youth: from diagnosis to treatment. Diabetol Metab Syndr 2, 55.

3. Halpern A \& Mancini MC (2003) Treatment of obesity: an update on anti-obesity medications. Obes Rev 4, 25-42.

4. Giugliano D, Ceriello A \& Esposito K (2008) Are there specific treatments for the metabolic syndrome? Am J Clin Nutr 87, 8-11.

5. Isomac B, Almgern P, Tuani T, et al. (2001) Cardiovascular morbidity and mortality associated with the metabolic syndrome. Diabetes Care 24, 683-689.

6. Weiss R (2010) Metabolic syndrome in childhood - causes and effects. Endocr Dev 19, 62-72.
7. Ford ES, Giles WH \& Dietz WH (2002) Prevalence of the metabolic syndrome among US adults: findings from the Third National Health and Nutrition Examination Survey. JAMA 287, 356-359.

8. Azizi F, Salehi P, Etemadi A, et al. (2003) Prevalence of metabolic syndrome in an urban population: Tehran Lipid and Glucose Study. Diabetes Res Clin Pract 61, 29-37.

9. Azadbakht L, Kimiagar M, Mehrabi Y, et al. (2007) Soy inclusion in the diet improves features of the metabolic syndrome: a randomized crossover study in postmenopausal women. Am J Clin Nutr 85, 735-741.

10. Mancini MC (2009) Metabolic syndrome in children and adolescents - criteria for diagnosis. Diabetol Metab Syndr 1, 20.

11. Esmaillzadeh A, Mirmiran P, Azadbakht L, et al. (2006) High prevalence of the metabolic syndrome in Iranian adolescents. Obesity 14, 377-382.

12. Grundy SM, Cleeman JI, Daniels RD, et al. (2005) Diagnosis and management of the metabolic syndrome: an American Heart Association/National Heart, Lung, and Blood Institute Scientific Statement. Circulation 112, 2735-2752.

13. Shenoy SF, Poston WSC, Reeves R, et al. (2010) Weight loss in individuals with metabolic syndrome given DASH diet counseling when provided a low sodium vegetable juice: a randomized controlled trial. Nutr $J \mathbf{9}, 8$.

14. Azadbakht L, Mirmiran P, Esmaillzadeh A, et al. (2005) Beneficial effects of a Dietary Approaches to Stop Hypertension eating plan on features of the metabolic syndrome. Diabetes Care 28, 2823-2831.

15. US Department of Health and Human Services (2006) Your Guide to Lowering Your Blood Pressure with DASH. Bethesda, MD: National Heart, Lung, and Blood Institute. (NIH Publication No. 06-4082).

16. Obarzanek E, Sacks FM, Vollmer WM, et al. (2001) Effects on blood lipids of a blood pressure-lowering diet: the Dietary Approaches to Stop Hypertension (DASH) Trial. Am J Clin Nutr 74, 80-89.

17. Moore TJ, Conlin PR, Ard J, et al. (2001) DASH (Dietary Approaches to Stop Hypertension) diet is effective treatment for stage 1 isolated systolic hypertension. Hypertension $\mathbf{3 8}$, 155-158.

18. Azadbakht L, Surkan PJ, Esmaillzadeh A, et al. (2011) The Dietary Approaches to Stop Hypertension eating plan affects C-reactive protein, coagulation abnormalities, and hepatic function tests among type 2 diabetic patients. J Nutr 141, 1083-1088.

19. Harnden KE, Frayn KN \& Hodson L (2010) Dietary Approaches to Stop Hypertension (DASH) diet: applicability and acceptability to a UK population. J Hum Nutr Diet 23, $3-10$.

20. Azadbakht L, Mirmiran P, Esmaillzadeh A, et al. (2005) Dairy consumption is inversely associated with the prevalence of the metabolic syndrome in Tehranian adults. Am J Clin Nutr 82, 523-530.

21. Al-Solaiman Y, Jesri A, Mountford WK, et al. (2010) DASH lowers blood pressure in obese hypertensives beyond potassium, magnesium and fibre. J Hum Hypertens 24 , 237-246.

22. Couch SC, Saelens BE, Levin L, et al. (2008) The efficacy of a clinic-based behavioral nutrition intervention emphasizing a DASH-type diet for adolescents with elevated blood pressure. J Pediatr 152, 494-501.

23. Wilson DK \& Ampey-Thornhill G (2001) The role of gender and family support on dietary compliance in an African American adolescent hypertension prevention study. Ann Behav Med 23, 59-67. 
24. Kelishadi R, Ardalan G, Gheiratmand R, et al. (2006) Paediatric metabolic syndrome and associated anthropometric indices: the CASPIAN Study. Acta Paediatr 95, 1625-1634.

25. American Dietetic Association, Pediatric Weight Management Guideline (2005) The 2005 US Institutes of Medicine "Dietary Reference Intakes for Energy, Carbohydrate, Fiber, Fat, Fatty Acids, Cholesterol, Protein, and Amino Acids (Macronutrients)". Washington, DC: National Academies Press. http:// www.adaevidencelibrary.com/topic.cfm?format_table$\mathrm{s}=0$ \&cat $=3060$ \&auth $=1$

26. Anonymous (2011) Healthy Eating Plate dishes out sound diet advice. More specific than My Plate, it pinpoints the healthiest food choices. Harv Heart Lett 22, 6.

27. Azadbakht L \& Esmaillzadeh A (2012) Macro and micronutrients intake, food groups consumption and dietary habits among female students in Isfahan University of Medical Sciences. Iran Red Crescent Med J 14, 204-209.

28. Pan Y \& Pratt CA (2008) Metabolic syndrome and its association with diet and physical activity in US adolescents. $J$ Am Diet Assoc 108, 276-286.

29. Carlson JJ, Eisenmann JC, Norman GJ, et al. (2011) Dietary fiber and nutrient density are inversely associated with the metabolic syndrome in US adolescents. J Am Diet Assoc 111, 1688-1695.

30. Liese AD, Bortsov A, Günther AL, et al. (2011) Association of DASH diet with cardiovascular risk factors in youth with diabetes mellitus: the SEARCH for Diabetes in Youth study. Circulation 123, 1410-1417.

31. Moore LL, Singer MR, Bradlee ML, et al. (2005) Intake of fruits, vegetables, and dairy products in early childhood and subsequent blood pressure change. Epidemiology 16, 4-11.

32. Azadbakht L, Fard NR, Karimi M, et al. (2011) Effects of the Dietary Approaches to Stop Hypertension (DASH) eating plan on cardiovascular risks among type 2 diabetic patients: a randomized crossover clinical trial. Diabetes Care 34, 55-57.

33. He FJ, Markandu ND, Coltart R, et al. (2005) Effect of shortterm supplementation of potassium chloride and potassium citrate on blood pressure in hypertensives. Hypertension 45, 571-574.

34. Streppel MT, Arends LR, van't Veer P, et al. (2005) Dietary fiber and blood pressure: a meta-analysis of randomized placebo-controlled trials. Arch Intern Med 9, 95-97.

35. Elliott P, Stamler J, Nichol R, et al. (1996) Intersalt revisited: further analyses of 24-hour sodium excretion and blood pressure within and across populations. Intersalt Cooperative Research Group. Br Med J 312, 1249-1253.

36. Dickinson HO, Nicolson DJ, Cook JV, et al. (2006) Calcium supplementation for the management of primary hypertension in adults. The Cochrane Database of Systematic Reviews 2006, issue 2 CD004639.

37. Bucher HC, Cook RJ, Guyatt GH, et al. (1996) Effects of dietary calcium supplementation on blood pressure: a metaanalysis of randomized controlled trials. JAMA 275, 1016-1022.

38. Esmaillzadeh A \& Azadbakht L (2008) Consumption of hydrogenated versus nonhydrogenated vegetable oils and risk of insulin resistance and the metabolic syndrome among Iranian adult women. Diabetes Care 31, 223-226.

39. Ward NC, Hodgson JM, Croft KD, et al. (2005) The combination of vitamin $\mathrm{C}$ and grape-seed polyphenols increases blood pressure: a randomized, double-blind, placebo-controlled trial. J Hypertens 23, 427-434.

40. D'Uscio LV, Milstein S, Richardson D, et al. (2003) Long-term vitamin $\mathrm{C}$ treatment increases vascular tetrahydrobiopterin levels and nitric oxide synthase activity. Circ Res 92, 88-95.

41. Van Etten RW, de Koning EJ, Verhaar MC, et al. (2002) Impaired NO-dependent vasodilation in patients with type II (non-insulin-dependent) diabetes mellitus is restored by acute administration of folate. Diabetologia 45, 1004-1010.

42. McEneny J, Couston C, McKibben B, et al. (2007) Folate: in vitro and in vivo effects on VLDL and LDL oxidation. Int J Vitam Nutr Res 77, 66-72.

43. Shikany JM, Phadke RP, Redden DT, et al. (2009) Effects of low- and high-glycemic index/glycemic load diets on coronary heart disease risk factors in overweight/obese men. Metabolism 58, 1793-1801. 